\title{
EXTREMAL PROBLEMS IN THE FOCK SPACE
}

\author{
CATHERINE BÉNÉTEAU, BRENT J. CARSWELL, AND SHERWIN KOUCHEKIAN
}

\begin{abstract}
This paper is devoted to examining some extremal problems in the Fock space. We discuss the order and type of Fock space functions and pose an extremal problem for a zero-based subspace corresponding to a finite zero set. We examine the zeros of the extremal function and solve an extremal problem for non-vanishing functions.
\end{abstract}

\section{INTRODUCTION}

The Fock space $F^{2}$ is the set of entire functions $f$ in the complex plane $\mathbb{C}$ with

$$
\|f\|_{2}^{2}:=\int_{\mathbb{C}}|f(z)|^{2} e^{-|z|^{2}} d A(z)<\infty
$$

where $d A(z)=\frac{1}{\pi} d x d y$ is normalized area measure. The space $F^{2}$ is a Hilbert space with inner product

$$
\langle f, g\rangle:=\int_{\mathbb{C}} f(z) \overline{g(z)} e^{-|z|^{2}} d A(z) .
$$

Using the power series expansion of an entire function $f(z)=\sum_{n=0}^{\infty} a_{n} z^{n}$ in $\mathbb{C}$, it not hard to show (see [2]) that the partial sums converge to $f$ in $F^{2}$. As a consequence, we have that the set of all polynomials is a dense linear manifold in the Fock space. Moreover, it follows that the sequence

$$
e_{n}(z)=\frac{z^{n}}{\sqrt{n !}}, \quad n=0,1,2, \ldots
$$

forms an orthonormal basis of $F^{2}$ (see [2]). Using (1.2), one can write the norm of a function in terms of its Taylor coefficients; that is, if $f(z)=\sum_{n=0}^{\infty} a_{n} z^{n}$, then

$$
\|f\|_{2}^{2}=\left\langle\sum_{n=0}^{\infty} a_{n} z^{n}, \sum_{m=0}^{\infty} a_{m} z^{m}\right\rangle=\sum_{n=0}^{\infty} n !\left|a_{n}\right|^{2} .
$$

2000 Mathematics Subject Classification. Primary: 30D15, Secondary: 30E05 .

Key words and phrases. Fock spaces, entire functions, extremal problems.

The first named author would like to thank the Internal Awards Program at USF for support during work on this project. The second named author would like to thank the Allegheny College Academic Support Committee for funding while this work was being developed. The third named author was supported in part by the National Science Foundation grant DMS0500916 . 
Note that, given the inner product (1.1), multiplication by $z$ and differentiation are adjoint operators (at least formally): that is, for any polynomials $p$ and $q$,

$$
\langle z p(z), q(z)\rangle=\left\langle p(z), q^{\prime}(z)\right\rangle .
$$

In fact, if we define an inner product as in (1.1) where $e^{-|z|^{2}}$ is replaced by any continuous, radial weight $\omega(z)$ and require multiplication by $z$ and differentiation to be adjoint operators in the sense defined by (1.4), then $\omega(z)$ must be equal to $e^{-|z|^{2}}$. (See [19] for details.)

Fischer defined this inner product in [7] and used it to study partial differential equations. The adjoint condition (1.4) turned out to be useful in defining certain "creation" and "annihilation" operators in quantum mechanics. Spaces related to $F^{2}$ were studied independently by various physicists, in particular Fock in [8] and Bargmann in [2], and since then, these spaces (called Fock spaces, Fischer spaces, Bargmann spaces, among other names) have been studied by several authors. In [15], Newman and Shapiro examined a certain duality between polynomial ideals and polynomial solutions of systems of partial differential equations with constant coefficients (see also [16]). Seip and Wallsteén (see [17, 18]) characterized sampling and interpolation sets for the Fock spaces. Tung wrote a Ph.D. thesis on Fock spaces and published various results on the coefficients of Fock space functions in $[20,21,22]$. The point of departure for our work is the paper [23], in which Zhu proved some general results regarding the zero sets of the Fock spaces $F_{\alpha}^{p}$.

This paper is organized as follows. In Section 2, we discuss restrictions on the order and type of functions belonging to the Fock space. In Section 3, we examine the zeros of the extremal functions for zero-based subspaces. In Section 4, we solve a Carathéodory type extremal problem for non-vanishing functions in the Fock space.

The authors would like to thank Dmitry Khavinson for several helpful remarks.

\section{Order And Type of Fock Space Functions}

Let us recall here some of the basic definitions associated with the growth of a (non-constant) entire function $f$. (See $[3,14]$ for a nice introduction to the topic.) Define, for $r>0$,

$$
M_{f}(r)=M(r):=\max \{|f(z)|:|z|=r\} .
$$

The function $M(r)$ is clearly an increasing function such that $\lim _{r \rightarrow \infty} M(r)=\infty$. Note that by Cauchy's inequalities, unless $f$ is a polynomial, $M(r)$ must grow faster than any power of $r$. Using the exponential function as a possible measure of growth leads to the following definitions.

Definition 2.1. The order $\rho$ of an entire function $f$ is defined to be

$$
\rho:=\limsup _{r \rightarrow \infty} \frac{\ln \ln M_{f}(r)}{\ln r} .
$$


Definition 2.2. Given an entire function $f$ of finite order $\rho$, the type $\sigma$ of the function is defined to be

$$
\sigma:=\limsup _{r \rightarrow \infty} \frac{\ln M_{f}(r)}{r^{\rho}}
$$

For example, the function $f(z)=e^{3 z^{2}}$ is of order 2 and type 3. An entire function can have any order $0 \leq \rho \leq \infty$, and if it has finite order $\rho$, it can have any type $0 \leq \sigma \leq \infty$. The following facts regarding the types and orders of functions in the Fock space are well known. We include the proofs for completeness.

Proposition 2.3. Let $f$ be an entire function in $\mathbb{C}$. Then the following hold.

(1) If $f$ is in the Fock space, then the order of $f$ is less than or equal to 2 and if $f$ has order 2, then its type must be less than or equal to 1/2.

(2) In addition, if $f$ has order strictly less than 2 , or order 2 and type strictly less than $1 / 2$, then $f$ is in the Fock space.

Proof. First notice that (2) is straightforward. Indeed, if $f$ has order $\rho<2$, then it follows that

$$
|f(z)| \leq C \exp \left(|z|^{2-\varepsilon}\right)
$$

for some constant $C$, some $\varepsilon>0$ and all $|z|>R$, for a large enough $R$, which is enough to ensure that $f \in F^{2}$. Also, if $f$ has order 2 and type $\sigma<1 / 2$, then

$$
|f(z)| \leq C \exp \left\{(1 / 2-\varepsilon)|z|^{2}\right\}
$$

for some constant $C$, some $\varepsilon>0$ and $|z|>R$ for some $R$, which again guarantees that $f \in F^{2}$.

To prove (1), let $f \in F^{2}$ have the power series expansion $f(z)=\sum_{n=0}^{\infty} a_{n} z^{n}$ in $\mathbb{C}$. Then by the Cauchy-Schwarz inequality and (1.3), we have

$$
|f(z)|^{2} \leq\left(\sum_{n=0}^{\infty}\left|a_{n}\right|\left|z^{n}\right|\right)^{2} \leq\left(\sum_{n=0}^{\infty} \frac{\left|z^{n}\right|^{2}}{n !}\right) \cdot\left(\sum_{n=0}^{\infty} n !\left|a_{n}\right|^{2}\right)=e^{|z|^{2}}\|f\|_{2}^{2} .
$$

Thus,

$$
|f(z)| \leq e^{\frac{1}{2}|z|^{2}}\|f\|_{2}
$$

Now the proof follows from the definitions of order and type given above.

The following example explains why the borderline case of type 2 and order $\frac{1}{2}$ is excluded from the statement of Proposition 2.3. Consider the function

$$
f(z)=\sum_{n=1}^{\infty} \frac{1}{n \sqrt{n !}} z^{n} .
$$

Then $\|f\|_{2}^{2}=\sum_{n=1}^{\infty} \frac{1}{n^{2}}<\infty$, so $f$ is clearly in the Fock space $F^{2}$. Let us examine the order and type of this function. It is well-known that the order and type of 
an entire function can be computed from its Taylor coefficients (see, for example, [14]). More specifically, an entire function $\sum_{n=0}^{\infty} a_{n} z^{n}$ has order

$$
\rho=\limsup _{n \rightarrow \infty} \frac{n \ln n}{\ln \frac{1}{\left|a_{n}\right|}}
$$

and type

$$
\sigma=\frac{1}{e \rho} \limsup _{n \rightarrow \infty} n\left|a_{n}\right|^{\rho / n}
$$

Notice that since the function $f$ in $(2.2)$ is in the Fock space $F^{2}, f$ has order less than or equal to 2 . On the other hand,

$$
\rho=\limsup _{n \rightarrow \infty} \frac{n \ln (n)}{\ln (n \sqrt{n !})} \geq \limsup _{n \rightarrow \infty} \frac{n \ln (n)}{\ln (n)+\frac{1}{2} n \ln (n)}=2 .
$$

Therefore, $f$ has order 2 . In addition,

$$
\sigma=\frac{1}{2 e} \limsup _{n \rightarrow \infty} n\left|\frac{1}{n \sqrt{n !}}\right|^{\frac{2}{n}}=\frac{1}{2} .
$$

Notice that multiplying an entire function by $z$ does not change its order or type. In particular, $z f(z)$ is a function of order 2 and type $1 / 2$. However, $z f(z)$ is not in the Fock space, since

$$
\|z f(z)\|_{2}^{2}=\sum_{n=1}^{\infty} \frac{(n+1) !}{n^{2} n !}=\sum_{n=1}^{\infty} \frac{n+1}{n^{2}}=\infty .
$$

This example simultaneously shows that the Fock space cannot be defined simply in terms of order and type and that multiplication by $z$ is not well-defined on the Fock space. (In fact, multiplication by $z$ on the Fock space is one of the interesting examples of unbounded subnormal operators; see the introduction in [12] and the given references therein.)

One could also consider a simpler example, namely,

$$
f(z)=\frac{\sin \left((1 / 2) z^{2}\right)}{z^{2}}
$$

which is clearly an entire function of order 2 and type $1 / 2$. When $|z|=r$ gets large, $|f(z)|^{2}$ grows like $\frac{e^{r^{2}}}{r^{4}}$, which implies $\int_{0}^{2 \pi} \int_{0}^{\infty}\left|f\left(r e^{i \theta}\right)\right|^{2} e^{-r^{2}} r d r d \theta<\infty$, so that $f \in F^{2}$. On the other hand, $z f(z)$ is not in $F^{2}$. Indeed, this follows easily from the fact that when $|z|=r$ gets large,

$$
|z f(z)|^{2} e^{-r^{2}} r \sim \frac{1}{r} .
$$

The above discussion shows that, from a certain point of view, the interesting functions in the Fock space are the ones of order 2 and type 1/2. In fact, these Fock space functions have infinitely many zeros, which we now prove.

Proposition 2.4. A function in the Fock space of order 2 and type $1 / 2$ must have infinitely many zeros. 
Proof. By the Hadamard factorization theorem, an entire function $f$ of order 2 and type $1 / 2$ with finitely many zeros has the form

$$
f(z)=P(z) e^{a z+(1 / 2) z^{2}},
$$

where $P$ is a polynomial, and $a$ is a constant. Then

$$
\int_{\mathbb{C}}|f(z)|^{2} e^{-|z|^{2}} d A(z)=\frac{1}{\pi} \iint_{\mathbb{R}^{2}}|P(z)|^{2} e^{2 \operatorname{Re}(a) x} e^{-2\left(\operatorname{Im}(a) y+y^{2}\right)} d x d y .
$$

Since $P$ is a polynomial, there are positive constants $C$ and $R$ such that $|P(z)| \geq$ $C$ for $|z| \geq R$. It follows that

$$
\int_{\mathbb{C}}|f(z)|^{2} e^{-|z|^{2}} d A(z) \geq \frac{C^{2}}{\pi}\left(\int_{\mathbb{R} \backslash[-R, R]} e^{2 \operatorname{Re}(a) x} d x\right) \cdot\left(\int_{\mathbb{R}} e^{-2\left(\operatorname{Im}(a) y+y^{2}\right)} d y\right) .
$$

Clearly, the first integral in the right side of the above inequality diverges while the second integral converges. Therefore $f$ cannot be in the Fock space.

\section{An Extremal Problem}

Inspired by results in the theory of invariant subspaces of the Bergman space $A^{p}$ which began with the seminal paper of Hedenmalm [10] for $p=2$, were extended by Duren, Khavinson, Shapiro, and Sundberg in $[4,5]$ for $p \neq 2$, were developed by many others, and have now appeared in two books $[6,11]$, we investigate here the extremal function for a zero-based subspace of the Fock space $F^{2}$ associated with a finite zero set.

Note that in the Hardy and Bergman spaces, extremal functions come up naturally in connection with closed $z$-invariant subspaces, that is, closed subspaces $M$ such that $z M \subset M$. In the Fock space setting however, if $M$ is a non-trivial closed subspace of $F^{2}$ and $f \in F^{2}$ satisfies $f M \subset M$, then one can show that $f$ must be a bounded function, and being entire, must thus be a constant. (See [9] for a more detailed discussion.) In particular, there are no closed $z$-invariant subspaces of the Fock space other than the trivial space. However, one can still examine the extremal functions.

Generally, if $N$ is any closed subspace of $F^{2}$, we consider the problem of maximizing $|f(0)|$ among all $f \in N$ with $\|f\|_{2}=1$. (For convenience, we make the standing assumption that there is a function $f \in N$ with $f(0) \neq 0$; otherwise, we maximize a suitable derivative at the origin.) A function $f$ which achieves this maximum is called an extremal function for $N$. As in the Bergman space theory (see [11, Prop 3.5]), one can show that such an extremal function exists, belongs to $N$, and is unique up to rotation by a unimodular constant. Henceforth, $G_{N}$ will denote the unique extremal function for $N$ with the property that $G_{N}(0)>0$.

An extremal function $G_{N}$ can be described in terms of the reproducing kernel for $N$. First we mention that is is well known that $F^{2}$ is a reproducing kernel 
Hilbert space with reproducing kernel $K(z, w)=e^{\bar{w} z}$. In other words, $K(\cdot, w)$ is a function in $F^{2}$ which satisfies $f(w)=\langle f, K(\cdot, w)\rangle$ for all $f \in F^{2}$ and all $w \in \mathbb{C}$. We remark that the existence (and uniqueness) of the kernel function follow from the Riesz representation theorem and the inequality (2.1), which shows that point evaluation is a bounded linear functional on $F^{2}$. The explicit formula for the kernel follows from the fact that the functions $e_{n}$ in (1.2) form an orthonormal basis for $F^{2}$. (We omit the details.)

A closed subspace $N$ of $F^{2}$ has its own reproducing kernel $K_{N}(z, w)$. Indeed, $K_{N}(\cdot, w)$ is the orthogonal projection of $K(\cdot, w)$ onto $N$, for each $w \in \mathbb{C}$. Using an argument similar to the one for the Bergman space, one can easily show that

$$
G_{N}(z)=\frac{K_{N}(z, 0)}{\sqrt{K_{N}(0,0)}} .
$$

For our setting, we will only consider subspaces $N$ of $F^{2}$ associated with a finite zero set. Indeed, if $A=\left\{a_{1}, a_{2}, \ldots, a_{n}\right\}$ is a finite set of distinct non-zero points in $\mathbb{C}$, let

$$
N_{A}=\left\{f \in F^{2}: f\left(a_{j}\right)=0,1 \leq j \leq n\right\} .
$$

In what follows, for notational simplicity, the kernel function $K_{N_{A}}$ and the extremal function $G_{N_{A}}$ will be abbreviated $K_{A}$ and $G_{A}$ respectively.

Proposition 3.1. Let $A=\left\{a_{1}, a_{2}, \ldots, a_{n}\right\}$ be a set of distinct non-zero points in $\mathbb{C}$. Then the extremal function $G_{A}$ for the zero-based subspace $N_{A}$ has infinitely many zeros.

Proof. By considering a certain dual problem and using the reproducing property of kernels (see, e.g., [6, p. 14, 120]), it can be shown that $G_{A}$ is a linear combination of the kernels $K(z, 0)=1$ and $K\left(z, a_{k}\right)=e^{\bar{a}_{k} z}$ for $k=1, \ldots, n$. Thus, there are constants $c_{0}, \ldots, c_{n}$ such that

$$
G_{A}(z)=c_{0}+\sum_{k=1}^{n} c_{k} e^{\bar{a}_{k} z} .
$$

Note that if $n=1$, it is obvious that $G_{A}$ has infinitely many zeros. So let us assume that $n>1$ and $G_{A}$ has only finitely many zeros. Then there exists a polynomial $P(z)$ such that $G_{A}(z) / P(z)$ is a non-vanishing entire function. It follows that there is an entire function $h$ such that

$$
G_{A}(z)=P(z) e^{h(z)} \text {. }
$$

Note that multiplication by polynomials does not change the order of an entire function. Also, the order of a finite sum of functions equals the maximum of the corresponding orders. Therefore, since each factor $e^{\bar{a}_{k} z}$ has order 1 , it follows from (3.2) that $G_{A}$ has order 1 . Therefore, we must have $h(z)=b z+c$, where $b \neq 0$. Now (3.2) and (3.3) imply

$$
c_{0} e^{-b z}+\sum_{k=1}^{n} c_{k} e^{\left(\bar{a}_{k}-b\right) z}=P(z) e^{c} .
$$


Without loss of generality, we may assume $b \neq a_{k}$ for any $k$; otherwise, one of the terms of $G_{A}(z) e^{-b z}$ is a constant and can be included in the polynomial $P$ on the right hand side of (3.4). Since $n>1$ and the $a_{k}$ are distinct, there is at least one exponential term on the left hand side of (3.4). Next, we differentiate (3.4) $m=\operatorname{deg}(P)+1$ times with respect to variable $z$ to obtain

$$
c_{0}(-b)^{m} e^{-b z}+\sum_{k=1}^{n} c_{k}\left(a_{k}-b\right)^{m} e^{\left(\overline{a_{k}}-b\right) z} \equiv 0 .
$$

By our assumption on $b$ and the hypothesis of the proposition, $a_{1}, a_{2}, \ldots, a_{n}, b$ are distinct non-zero complex numbers. It follows that the exponential functions in (3.5) are linearly independent (see, for example, [14]). (Indeed, if $\alpha_{1}, \alpha_{2}, \ldots, \alpha_{n}$ are complex numbers, then the Wronskian of the functions $e^{\alpha_{k} z}, 1 \leq k \leq n$, at any point $z$ equals

$$
\prod_{i=1}^{n}\left|e^{\alpha_{i}}\right| \operatorname{det}(V),
$$

where $V$ is the square Vandermonde matrix, and

$$
\operatorname{det}(V)=\prod_{1 \leq i<j \leq n}\left(\alpha_{j}-\alpha_{i}\right)
$$

If the $\alpha_{k}$ are distinct, then the above determinant is non-zero, and thus the functions $e^{\alpha_{k} z}$ are linearly independent.)

Therefore equation (3.5) holds if and only if all the coefficients are zero. Since the coefficients $(-b)^{m}$ and $\left(a_{k}-b\right)^{m}$ are not zero, this forces $c_{k}=0$ for all $k$, which is not possible since $G_{A}$ is not identically zero.

The fact that these extremal functions have infinitely many more zeros than are prescribed is not that surprising because their building blocks are exponential functions. However, this is an interesting distinction between extremal functions in the Fock space and extremal functions in the Hardy or Bergman spaces, which vanish precisely on the prescribed zero set. In fact, this property of having no extraneous zeros is the key in the Hardy and Bergman spaces to getting isometric (for the Hardy spaces) or contractive (for the Bergman spaces) divisors. Therefore the fact that the Fock space extremal functions have many additional zeros eliminates the possibility of having contractive divisors in $F^{2}$. (See [23] for a more detailed discussion.) Finally, notice that these extremal functions are all of order 1 , and consequently the fact that they have infinitely many zeros could not have been deduced from Proposition 2.4.

In general, it is difficult to come up with exact formulas for the solutions to extremal problems; however, in the case of extremal functions with finitely many prescribed zeros, there is a nice iterative formula (see [11, p. 58]) to calculate the reproducing kernel $K_{A}$ of $N_{A}$, and thus the extremal function $G_{A}$. This procedure is based on adding one point at a time; that is, if one knows the reproducing kernel $K_{A}$ for $A=\left\{a_{1}, \ldots, a_{n}\right\}$, where $a_{1}, \ldots, a_{n}$ are distinct non-zero complex 
numbers, and if $a \notin A$, then

$$
K_{A \cup\{a\}}(z, w)=K_{A}(z, w)-\frac{K_{A}(z, a) K_{A}(a, w)}{K_{A}(a, a)} .
$$

Indeed, for each fixed $w \in \mathbb{C}$, the function on the right hand side of (3.6) represents a Fock space function of $z$ which vanishes whenever $z=a$ or $z=a_{i}$, $i=1,2, \ldots, n$, and therefore is in $N_{A \cup\{a\}}$. Moreover if $f \in N_{A \cup\{a\}}$, then $f \in N_{A}$ and $f(a)=0$, so

$$
\left\langle f, K_{A}(\cdot, w)-\frac{K_{A}(\cdot, a) K_{A}(a, w)}{K_{A}(a, a)}\right\rangle=f(w)-\frac{K_{A}(a, w)}{K_{A}(a, a)} f(a)=f(w) .
$$

By uniqueness of reproducing kernels, the formula (3.6) holds. Let us now use this iterative formula to compute the two point extremal function.

Proposition 3.2. Let $a$ and $b$ be distinct non-zero complex numbers. Then the two point extremal function is given by

$G_{\{a, b\}}(z)=C \frac{\left(e^{|a|^{2}}-e^{z \bar{a}}\right)\left(e^{|b|^{2}} e^{|a|^{2}}-e^{b \bar{a}} e^{a \bar{b}}\right)-\left(e^{z \bar{b}} e^{|a|^{2}}-e^{z \bar{a}} e^{a \bar{b}}\right)\left(e^{|a|^{2}}-e^{b \bar{a}}\right)}{\sqrt{\left(e^{|a|^{2}}-1\right)\left(e^{|b|^{2}} e^{|a|^{2}}-e^{b \bar{a}} e^{a \bar{b}}\right)-\left(e^{|a|^{2}}-e^{a \bar{b}}\right)\left(e^{|a|^{2}}-e^{b \bar{a}}\right)}}$,

where $C=\sqrt{e^{-|a|^{2}} /\left(e^{|b|^{2}} e^{|a|^{2}}-e^{b \bar{a}} e^{a \bar{b}}\right)}$.

Proof. In view of (3.1), we need to calculate $K_{\{a, b\}}$. We will achieve this iteratively using (3.6). First note that for the empty set, $N_{\emptyset}=F^{2}$, and

$$
K_{\emptyset}(z, w)=K(z, w)=e^{\bar{w} z} .
$$

Next let $A=\emptyset$ in (3.6) to get

$$
K_{\{a\}}(z, w)=e^{-|a|^{2}}\left(e^{z \bar{w}} e^{|a|^{2}}-e^{z \bar{a}} e^{a \bar{w}}\right) .
$$

Given $K_{\{a\}}$, another application of (3.6) yields

$$
\begin{aligned}
& K_{\{a, b\}}(z, w)= \\
& e^{-|a|^{2}}\left(e^{z \bar{w}} e^{|a|^{2}}-e^{z \bar{a}} e^{a \bar{w}}\right)-\frac{e^{-2|a|^{2}}\left(e^{z \bar{b}} e^{|a|^{2}}-e^{z \bar{a}} e^{a \bar{b}}\right)\left(e^{b \bar{w}} e^{|a|^{2}}-e^{b \bar{a}} e^{a \bar{w}}\right)}{e^{-|a|^{2}}\left(e^{|b|^{2}} e^{|a|^{2}}-e^{b \bar{a}} e^{a \bar{b}}\right)} .
\end{aligned}
$$

Rewriting the above equality implies

$$
\begin{aligned}
& K_{\{a, b\}}(z, w)=\frac{e^{-|a|^{2}}}{e^{|b|^{2}} e^{|a|^{2}}-e^{b \bar{a}} e^{a \bar{b}}} \times \\
& {\left[\left(e^{z \bar{w}} e^{|a|^{2}}-e^{z \bar{a}} e^{a \bar{w}}\right)\left(e^{|b|^{2}} e^{|a|^{2}}-e^{b \bar{a}} e^{a \bar{b}}\right)-\left(e^{z \bar{b}} e^{|a|^{2}}-e^{z \bar{a}} e^{a \bar{b}}\right)\left(e^{b \bar{w}} e^{|a|^{2}}-e^{b \bar{a}} e^{a \bar{w}}\right)\right] .}
\end{aligned}
$$

The result now follows from (3.1).

Notice that the (known) formula for the single point extremal function (see, e.g., [23]) falls out naturally from the above proof. 
Corollary 3.3. If $a \neq 0$, then the single point extremal function is given by

$$
G_{\{a\}}(z)=\frac{1-e^{\bar{a}(z-a)}}{\sqrt{1-e^{-|a|^{2}}}} .
$$

Proof. Combine (3.7) with (3.1).

In the remainder of this section, we present some results about the zeros of these extremal functions. First of all, it follows easily from Corollary 3.3 that that the zeros of the single point extremal functions $G_{\{a\}}$ are given by $z_{k}=a+2 \pi i k / \bar{a}$, where $k$ is an integer. For the two point extremal functions $G_{\{a, b\}}$, the situation is more complicated. We start with a basic lemma. Recall that $\mathbb{Q}$ denotes the set of rational numbers.

Lemma 3.4. Suppose $a, b$ are two distinct complex numbers such that $\frac{b}{a}=r$ belongs to $\mathbb{Q} \backslash\{0\}$, and let

$$
A=e^{|a|^{2}}, \quad \alpha=\frac{A^{r^{2}}-A^{r}}{A^{r}-A}, \quad \text { and } \quad \beta=\frac{A^{r^{2}+1}-A^{2 r}}{A^{r}-A} .
$$

(a) If $r=\frac{q}{p}$, where $q>p>0$ and $p$ and $q$ are relatively prime, then $z$ is a solution to $G_{\{a, b\}}(z)=0$ if and only if

$$
w^{q}-\alpha w^{p}+\beta=0,
$$

where $w=e^{\bar{a} z / p}$. Moreover, the polynomial equation (3.9) has exactly $q$ distinct solutions.

(b) If $r=-\frac{q}{p}$, where $q \geq p>0$ and $p$ and $q$ are relatively prime, then $z$ is a solution to $G_{\{a, b\}}(z)=0$ if and only if

$$
w^{p+q}+\beta w^{p}-\alpha=0,
$$

where $w=e^{-\bar{a} z / p}$. Moreover, the polynomial equation (3.10) has exactly $p+q$ distinct solutions.

Proof. From Proposition 3.2, we have $G_{\{a, b\}}(z)=0$ if and only if

$$
\left(e^{|a|^{2}}-e^{z \bar{a}}\right)\left(e^{\left(r^{2}+1\right)|a|^{2}}-e^{2 r|a|^{2}}\right)-\left(e^{z \bar{a} r} e^{|a|^{2}}-e^{z \bar{a}} e^{r|a|^{2}}\right)\left(e^{|a|^{2}}-e^{r|a|^{2}}\right)=0 .
$$

Collecting terms and using the notation in (3.8), we get that

$$
\left(-A^{2}+A^{r+1}\right) e^{z \bar{a} r}+e^{z \bar{a}}\left(-A^{r^{2}+1}+A^{r+1}\right)+\left(A\left(A^{r^{2}+1}-A^{2 r}\right)\right)=0 .
$$

Writing $w=e^{\bar{a} z / p}$ for $r=q / p$ and $w=e^{-\bar{a} z / p}$ for $r=-q / p$, a straightforward calculation leads to (3.9) and (3.10), respectively. Conversely, note that since $\beta \neq 0$, if $w$ is any solution to (3.9), then any $z$ satisfying $w=e^{\bar{a} z / p}$ is a solution to $G_{\{a, b\}}(z)=0$. A similar argument holds for (3.10).

Next, we prove that equation (3.9) has exactly $q$ solutions. (The proof that equation (3.10) has exactly $p+q$ solutions follows the same steps as those given for (3.9), and is therefore omitted.) To accomplish this, it is enough to show 
that any zero of (3.9) with multiplicity greater than one, if any, must be real, and that the real zeros of (3.9) are distinct.

So let $f(w)=w^{q}-\alpha w^{p}+\beta$ and assume that $w_{0}$ is a zero of $f$ with multiplicity greater than one (clearly $w_{0} \neq 0$ ). Thus, both $f$ and $f^{\prime}$ must vanish at $w_{0}$; that is,

$$
w_{0}^{q}-\alpha w_{0}^{p}+\beta=0 \quad \text { and } \quad q w_{0}^{q}-\alpha p w_{0}^{p}=0 .
$$

Solving the above system for $w_{0}^{p}$ and $w_{0}^{q}$ in terms of $\alpha$ and $\beta$ yields

$$
w_{0}^{p}=\frac{\beta}{\alpha(1-p / q)}>0 \quad \text { and } \quad w_{0}^{q}=\frac{\beta}{q / p-1}>0
$$

since it is easily seen that $\alpha$ and $\beta$ are positive. Using the polar form $w_{0}=r_{0} e^{i \theta_{0}}$, the first equality in (3.12) gives $\theta_{0}=2 \pi n / p$ for some integer $n$. Now, the second equality in (3.12) forces $q n / p$ to be an integer. Since $p$ and $q$ are relatively prime, we must have that $p$ divides $n$, which implies $\theta_{0}$ is multiple of $2 \pi$; i.e, $w_{0}$ is real.

It remains to show that the real roots of (3.9) are distinct. In fact, it will be shown that for $q$ an even number, equation (3.9) has exactly 2 distinct real roots; and for $q$ an odd number, (3.9) has exactly 3 distinct real roots. Since $G_{\{a, b\}}$ vanishes at $a$ and $b,(3.9)$ has at least two positive roots $x_{1}=e^{|a|^{2} / p}$ and $x_{2}=e^{r|a|^{2} / p}$.

Suppose $q=2 n$ and $p=2 m+1$, where $n>m \geq 0$, and let $f(x)=x^{2 n}-\alpha x^{2 m+1}+$ $\beta$. The critical points are solutions to $f^{\prime}(x)=x^{2 m}\left(2 n x^{2(n-m)-1}-(2 m+1) \alpha\right)=$ 0 , which are clearly 0 (unless $m=0$ ) and $x_{0}=\sqrt[N]{\alpha(2 m+1) /(2 n)}>0$, where $N=2(n-m)-1$. An easy calculation shows that $f^{\prime}(x)<0$ on $(-\infty, 0) \cup\left(0, x_{0}\right)$ and $f^{\prime}(x)>0$ on $\left(x_{0}, \infty\right)$. Consequently, $f$ has at most two zeros. But we already know that $x_{1}$ and $x_{2}$ are among the zeros of $f$. So the equation (3.9) has exactly two distinct real zeros. This proves part (a) for the case of $q$ being an even number.

Next, let $q=2 n+1, n \geq 1$, and $p=2 m, m \geq 1$. With $f(x)=x^{2 n+1}-\alpha x^{2 m}+\beta$, we have $f^{\prime}(x)=x^{2 m-1}\left(2 n x^{2(n-m)+1}-2 m \alpha\right)$. The critical points of $f$ are 0 and $x_{0}=\sqrt[N]{2 \alpha m /(2 n+1)}>0$, where $N=2(n-m)+1$. It follows that $f^{\prime}(x)>0$ on $(-\infty, 0), f^{\prime}(x)<0$ on $\left(0, x_{0}\right)$, and $f^{\prime}(x)>0$ on $\left(x_{0}, \infty\right)$. Therefore $f$ has at most 3 zeros, one of which must be negative, since $\beta>0$. Since $x_{1}$ and $x_{2}$ are both positive real zeros, $f$ and therefore (3.9) have exactly three distinct real roots. The proof of the final case where both $q$ and $p$ are odd numbers follows from a similar argument and will be omitted.

Noting that both $\alpha$ and $\beta$ are negative numbers for $r<0$, a similar argument as the one given above shows that the equation (3.10) has exactly 3 real solutions when $p+q$ is odd and 2 real solutions when $p+q$ is even.

Next we describe the zero set of the two point extremal function $G_{\{a, b\}}$ under some restrictions on the relative location of $a$ and $b$. In what follows, let $K_{\tau}$ represent the kernel $K(\cdot, \tau)$, where again $K(z, \tau)=e^{\bar{\tau} z}$. 
Theorem 3.5. Suppose $a, b$ are distinct complex numbers such that $\frac{b}{a}=r$ belongs to $\mathbb{Q} \backslash\{0\}$. Then there exists a number $\tau$ and a finite number of distinct points $w_{1}, w_{2}, \ldots, w_{m} \in \mathbb{C}$ for which the union of the level sets $K_{\tau}^{-1}\left(w_{i}\right), 1 \leq i \leq m$, coincides with the zero set of the extremal function $G_{\{a, b\}}$. Each level set $K_{\tau}^{-1}\left(w_{i}\right)$ consists of an infinite number of points which are all contained in a single line perpendicular to the line passing through $a$ and $b$. Moreover, the number $m$ of level sets, the number $\tau$, and the number $n$ of distinct lines containing the level sets satisfy the following:

(1) If $r=q / p$, where $p, q \in \mathbb{N}$ are relatively prime and $q>p>0$, then $m=q$, $\tau=a / p$, and

$$
\begin{cases}3 \leq n \leq 3+\frac{q-3}{2} & \text { if } q \text { is odd } \\ 2 \leq n \leq 2+\frac{q-2}{2} & \text { if } q \text { is even }\end{cases}
$$

(2) If $r=-q / p$, where $p, q \in \mathbb{N}$ are relatively prime and $q \geq p>0$, then $m=p+q, \tau=-a / p$, and

$$
\begin{cases}3 \leq n \leq 3+\frac{q+p-3}{2} & \text { if } q+p \text { is odd } \\ 2 \leq n \leq 2+\frac{q+p-2}{2} & \text { if } q+p \text { is even. }\end{cases}
$$

Remark. Notice that if the ratio $|b / a|<1$, then $|a / b|>1$, and thus Theorem 3.5 completely describes the zero set of the two point extremal function $G_{\{a, b\}}$ whenever $b / a$ is a non-zero rational number.

Proof. Let $r=q / p$, where $p$ and $q$ are relatively prime integers with $q>p>0$. (We will only handle the case $r>0$ because the case $r<0$ is similar and so the proof will be omitted.)

By Lemma 3.4, $z_{0}$ is a zero of $G_{\{a, b\}}$ if and only if $w_{0}$ is a root of the polynomial (3.9), where $w_{0}=e^{\bar{a} z_{0} / p}$. Said differently, if $w_{1}, w_{2}, \ldots, w_{q}$ are the $q$ distinct roots of (3.9) (we know there are exactly $q$ of them by Lemma 3.4), then any zero of $G_{\{a, b\}}$ must belong to a level set $K_{\tau}^{-1}\left(w_{j}\right)$, where $\tau=a / p$, for some $j=1,2, \ldots, q$, and conversely, any point in $K_{\tau}^{-1}\left(w_{j}\right)$ must be a zero of $G_{\{a, b\}}$.

Clearly, $K_{\tau}^{-1}\left(w_{j}\right)$ is comprised of the following points:

$$
z_{j}+2 k \pi i p / \bar{a}, \quad k \in \mathbb{Z},
$$

where $z_{j}=p / \bar{a} \ln \left|w_{j}\right|+i p / \bar{a} \arg \left(w_{j}\right)$, and where $\arg \left(w_{j}\right)$ is the principal value of the argument of $w_{j}$. Moreover, since $2 k \pi i p / \bar{a}=t i a$ where $t=2 k \pi p /|a|^{2}$ is real, it is clear that for a fixed $j$, the points in (3.13) all lie on a single line perpendicular to the line through $a$ and $b$. (The perpendicularity is apparent since $a, b$, and 0 are colinear due to the hypothesis that $b / a$ is real.)

The proof is now complete, provided we can establish the restriction on the number $n$ of distinct lines.

Before doing so, we first claim that for $|\lambda|=1$, the point $z_{0}$ is a zero of $G_{\{a, b\}}$ if and only if $\lambda z_{0}$ is a zero of $G_{\{\lambda a, \lambda b\}}$. To prove the claim, notice that since $\lambda \neq 0$, it is enough to prove one direction of the equivalence. So, suppose $G_{\{a, b\}}=0$. 
Then, by Lemma $3.4, w_{0}=e^{\bar{a} z_{0} / p}$ is a root of the equation $w^{q}-\alpha w^{p}+\beta=0$, where $\alpha$ and $\beta$ are as in (3.8). Notice that since $|\lambda|=1$, the values of $\alpha$ and $\beta$ in (3.8) remain unchanged if $a$ and $b$ are replaced with $\lambda a$ and $\lambda b$. Since $w_{0}=e^{\overline{\lambda a}\left(\lambda z_{0}\right) / p}$, it follows from Lemma 3.4 that $\lambda z_{0}$ is a zero of $G_{\{\lambda a, \lambda b\}}$. (Notice that Lemma 3.4 can be applied in the case of the two points $\lambda a$ and $\lambda b$, since $\lambda b /(\lambda a)=r=q / p \in \mathbb{Q} \backslash\{0\}$.$) This proves the claim.$

As a result of the claim, the number of distinct lines remains invariant if the zeros are rotated. Thus, from now on, we will assume that $a$ and $b$ are real.

Next, we obtain the upper bound on $n$. Observe that since $\alpha$ and $\beta$ are real, the non-real roots of (3.9) must appear in conjugate pairs. We claim that if $w_{0}$ and $\overline{w_{0}}$ are conjugate roots of $(3.9)$, then $K_{\tau}^{-1}\left(w_{0}\right)$ and $K_{\tau}^{-1}\left(\overline{w_{0}}\right)$ are contained in the same line perpendicular to the real axis. (Recall that $\tau=a / p$.) Indeed, if $z_{0}$ satisfies $e^{z_{0}}=w_{0}$, then $e^{\overline{z_{0}}}=\overline{w_{0}}$. By (3.13), points in $K_{\tau}^{-1}\left(w_{0}\right)$ all have the same real part as $z_{0}$, while points in $K_{\tau}^{-1}\left(\overline{w_{0}}\right)$ all have the same real part as $\overline{z_{0}}$. Since $\operatorname{Re}\left(z_{0}\right)=\operatorname{Re}\left(\overline{z_{0}}\right)$, the claim is now apparent.

From the proof of Lemma 3.4, we know that if $q$ is odd, then (3.9) has exactly 3 distinct real roots, and consequently the zero set of $G_{\{a, b\}}$ is contained in at most $3+(q-3) / 2$ distinct lines, 3 corresponding to the level sets of the real roots of (3.9) and $(q-3) / 2$ corresponding to the level sets of the $q-3$ non-real roots of (3.9). Arguing similarly for even $q$ establishes the desired upper bound on $n$.

To obtain the lower bound, we first observe that if $w_{1}$ and $w_{2}$ are roots of (3.9) where $K_{\tau}^{-1}\left(w_{1}\right)$ and $K_{\tau}^{-1}\left(w_{2}\right)$ lie on the same line perpendicular to the real axis, then $\left|w_{1}\right|=\left|w_{2}\right|$. (To see this, notice that if $z_{j} \in K_{\tau}^{-1}\left(w_{j}\right)$ for $k=1,2$, then by (3.13), we have $\operatorname{Re}\left(z_{1}\right)=\operatorname{Re}\left(z_{2}\right)$, which easily yields $\left.\left|w_{1}\right|=\left|w_{2}\right|.\right)$

The lower bound for the case of $q$ even is now clear. Indeed, the proof of Lemma 3.4 shows that when $q$ is even, (3.9) has two real roots, namely $w_{1}=e^{a^{2}}$ and $w_{2}=e^{b^{2}}$. Since $a^{2} \neq b^{2}$, we have $\left|w_{1}\right| \neq\left|w_{2}\right|$, and so $K_{\tau}^{-1}\left(w_{1}\right)$ and $K_{\tau}^{-1}\left(w_{2}\right)$ are contained in different lines.

Finally, suppose that $q$ is odd. By the proof of Lemma 3.4, equation (3.9) has exactly 3 real roots, namely $w_{1}=e^{a^{2}}, w_{2}=e^{b^{2}}$, and some negative number $w_{3}$. If we can show that $\left|w_{3}\right| \neq w_{1}$ and $\left|w_{3}\right| \neq w_{2}$, then the result follows from the observation above. To this end, assume that there are real numbers $w$ and $-w$ satisfying (3.9). Noting that $q$ is odd, we must have that

$$
\left\{\begin{array}{l}
w^{q}-\alpha w^{p}+\beta=0 \\
-w^{q}-(-1)^{p} \alpha w^{p}+\beta=0 .
\end{array}\right.
$$

The above equations imply that either $\beta=0$ (for $p$ odd) or $w=0$ (for $p$ even) which cannot happen. This completes the proof.

Theorem 3.5 shows that the extremal function has many zeros in addition to the two prescribed, and the additional zeros appear in a surprising nontrivial way. In other words, while we expect the additional zeros due to the periodicity of the exponential function, we also see that completely new level sets appear in 
the zeros of the extremal function. This implies in particular that there is no hope for an isometric or contractive divisor as we have in a Hardy or Bergman space situation (see also [23] for a discussion of this fact). Finally, by disturbing the original zeros slightly, we completely change the structure of the zeros of the extremal function, since the ratio of the two zeros can become real, or the zeros may no longer lie on the same line through the origin, for example. We do not know the exact structure of the zero set of the extremal function when the ratio of the zeros is irrational or non-real. In that case, the transcendental equation (3.11) can no longer be transformed into a polynomial equation. This type of equation has been studied in [13], and it may be possible to extract precise information in our setting from the results in that paper.

It should be mentioned that all upper bounds for $n$ given by Theorem 3.5 can be achieved. For instance, taking $a=1$ and $b=4$, one can show that

$$
w^{4}-\alpha w+\beta=(w-e)\left(w-e^{4}\right)\left(w^{2}-\left(e+e^{4}\right) w+e^{2}+e^{5}+e^{8}\right) .
$$

Denoting by $w_{3}$ and $\overline{w_{3}}$ the pair of conjugate roots corresponding to the last factor of the above equality, it follows that $\left|w_{3}\right|=\sqrt{e^{2}+e^{5}+e^{8}}$, which is strictly larger than the two roots $e$ and $e^{4}$. Thus, there are 4 level sets $K_{\tau}^{-1}\left(w_{1}\right)$ contained in exactly 3 distinct lines in this case. On the other hand, if $a=1$ and $b=2$ or $b=3$, then the lower bound and the upper bound in part (1) of Theorem 3.5 are equal, and thus are trivially attained. Similarly, if $a=-1$ and $b=1$ or $b=2$, the lower bound and the upper bound in part (2) of Theorem 3.5 are equal and thus are trivially attained. In fact, we conjecture that the number $n$ of distinct lines containing the level sets is always equal to the relevant upper bound given in Theorem 3.5. In order to see why this might be true, recall that in the proof of Theorem 3.5, it was shown (in the case that $r>0$, for example) that if two level sets lie on the same line then the corresponding roots of the equation (3.9) have the same modulus. To see the difficulty here, suppose $w$ and $\lambda w$, where $|\lambda|=1$, are roots of (3.9). Then after some straightforward algebraic manipulation, one can show that $\lambda$ has to satisfy the following non-linear equation.

$$
\left\{\begin{array}{l}
\alpha\left(1-\lambda^{p}\right)^{q}=\left(1-\lambda^{q}\right)^{p} \\
|\lambda|=1
\end{array}\right.
$$

Note that (3.14) has already the two trivial solutions $\lambda=1$ and $\lambda=\bar{w} / w$ corresponding to the solutions $w$ and $\bar{w}$ of (3.9), respectively. If these are the only solutions to (3.14), then $n$ is equal to the upper bound in Theorem 3.5.

\section{An Extremal Problem for Non-Vanishing Functions}

Let us now turn to a discussion of a Carathéodory type extremal problem for non-vanishing functions in $F^{2}$. Notice that if $f$ is a non-vanishing function in $F^{2}$, then the Hadamard factorization of $f$, together with Propositions 2.3 and 2.4, imply that there exist constants $a, b, c$, with $|a|<1 / 2$, such that

$$
f(z)=e^{a z^{2}+b z+c} .
$$


Now, fix $A>0$ and consider the extremal problem of finding

$$
\inf \left\{\|f\|_{2}: f(0)=1, f^{\prime}(0)=A, f \text { non-vanishing in } \mathbb{C}\right\} .
$$

By a standard normal family argument and the Cauchy-Schwarz inequality, it is easy to show that the extremal function $f_{*}$ exists and is unique. (See, for example, [1] for the same argument in Bergman spaces.) The interpolating conditions immediately imply that $c=0$ and therefore $f_{*}$ has the form $f_{*}(z)=e^{a z^{2}+A z}$, where $|a|<1 / 2$.

Lemma 4.1. The Maclaurin series for the extremal function $f_{*}$ has real coefficients. Consequently, the constant a in the factorization of $f_{*}$ must be real.

Proof. Notice that the function $\overline{f_{*}(\bar{z})}$ is entire, has the same norm as $f_{*}$, and satisfies the interpolating conditions. Therefore, by uniqueness of the extremal function, $\overline{f_{*}(\bar{z})} \equiv f_{*}(z)$. This immediately implies that the coefficients of $f_{*}$ must be real. In particular, since $A$ is real and $f_{*}^{\prime \prime}(0)=2 a+A^{2}$, it follows that $a$ is real.

Let us now calculate the norm of a function $f(z)=e^{a z^{2}+A z}$, for $A>0$ and $a$ real-valued. Observe first that

$$
\begin{aligned}
\|f\|_{2}^{2} & =\int_{\mathbb{C}}\left|e^{a z^{2}+A z}\right|^{2} e^{-|z|^{2}} d A(z) \\
& =\int_{-\infty}^{\infty} \int_{-\infty}^{\infty} \exp \left(2 \operatorname{Re}\left[a(x+i y)^{2}+A(x+i y)\right]-x^{2}-y^{2}\right) \frac{d x d y}{\pi} \\
& =\frac{1}{\pi} \int_{-\infty}^{\infty} \exp \left(-(1-2 a) x^{2}+2 A x\right) d x \int_{-\infty}^{\infty} \exp \left(-(1+2 a) y^{2}\right) d y
\end{aligned}
$$

Notice that since $-\frac{1}{2}<a<\frac{1}{2}$, these integrals converge. Now, by a simple change of variables, the second integral is easily seen to be equal to

$$
\frac{1}{\sqrt{2 a+1}} \int_{-\infty}^{\infty} e^{-y^{2}} d y=\frac{\sqrt{\pi}}{\sqrt{2 a+1}}
$$

On the other hand, by completing the square and by a change of variables, the first integral can be rewritten as follows:

$$
\begin{aligned}
& \int_{-\infty}^{\infty} \exp \left(-(1-2 a) x^{2}+2 A x\right) d x \\
= & \int_{-\infty}^{\infty} \exp \left(-(1-2 a)\left[\left(x-\frac{A}{1-2 a}\right)^{2}-\left(\frac{A}{1-2 a}\right)^{2}\right]\right) d x \\
= & \exp \left(\frac{A^{2}}{1-2 a}\right) \int_{-\infty}^{\infty} \exp \left(-(1-2 a)\left(x-\frac{A}{1-2 a}\right)^{2}\right) d x \\
= & \exp \left(\frac{A^{2}}{1-2 a}\right) \int_{-\infty}^{\infty} \exp \left(-(1-2 a) x^{2}\right) d x \\
= & \exp \left(\frac{A^{2}}{1-2 a}\right) \frac{\sqrt{\pi}}{\sqrt{1-2 a}}
\end{aligned}
$$


Putting both calculations together gives

$$
\|f\|_{2}^{2}=\frac{1}{\pi} \frac{\sqrt{\pi}}{\sqrt{2 a+1}} \exp \left(\frac{A^{2}}{1-2 a}\right) \frac{\sqrt{\pi}}{\sqrt{1-2 a}}=\frac{\exp \left(A^{2} /(1-2 a)\right)}{\sqrt{1-4 a^{2}}} .
$$

Therefore, to solve Problem (4.1), we need to find $a$ which minimizes the function

$$
g(a):=\frac{\exp \left(A^{2} /(1-2 a)\right)}{\sqrt{1-4 a^{2}}}
$$

for $-\frac{1}{2}<a<\frac{1}{2}$, where $A>0$ is fixed. A direct calculation shows that

$$
g^{\prime}(a)=2 \exp \left(\frac{A^{2}}{1-2 a}\right) \frac{1}{\left(1-4 a^{2}\right)^{3 / 2}(1-2 a)}\left(-4 a^{2}+2\left(A^{2}+1\right) a+A^{2}\right) .
$$

Therefore the sign of $g^{\prime}(a)$ depends on the sign of the quadratic polynomial $p(a)=-4 a^{2}+2\left(A^{2}+1\right) a+A^{2}$. The roots of $p$ are at

$$
c_{0}=\frac{1+A^{2}-\sqrt{A^{4}+6 A^{2}+1}}{4} \quad \text { and } \quad c_{1}=\frac{1+A^{2}+\sqrt{A^{4}+6 A^{2}+1}}{4} .
$$

It is not hard to see that $-\frac{1}{2}<c_{0}<0$ and $c_{1}>\frac{1}{2}$. Therefore $p(a) \geq 0$ for $c_{0} \leq a<\frac{1}{2}$, and $p(a) \leq 0$ for $-\frac{1}{2}<a \leq c_{0}$, and thus, the function $g$ achieves its minimum at $c_{0}$. This leads us to the following.

Theorem 4.2. The unique solution to the extremal problem

$$
\inf \left\{\|f\|_{2}: f(0)=1, f^{\prime}(0)=A, f \text { non-vanishing in } \mathbb{C}\right\}
$$

is the function

$$
f_{*}(z)=\exp \left(-a z^{2}+A z\right)
$$

where $a$ is the positive constant $\left(\sqrt{A^{4}+6 A^{2}+1}-\left(1+A^{2}\right)\right) / 4$.

Recall from Proposition 2.4 that a non-vanishing function in the Fock space cannot be of order 2 and of type exactly $1 / 2$; however, it can be of order 2 and type (less than and) arbitrarily close to $1 / 2$. Since the constant $a$ from Theorem 4.2 approaches $1 / 2$ from below as $A \rightarrow \infty$, the non-vanishing extremals from the above theorem provide such an example.

Finally, one can define $F^{p}$ to be the space of entire functions $f$ such that

$$
\int_{\mathbb{C}}|f(z)|^{p} e^{-|z|^{2}} d A(z)<\infty
$$

Then Theorem 4.2 can easily be used to give a solution to the same problem in $F^{p}$, for $0<p<\infty$, since given any non-vanishing function $f$ in $F^{p}$ that satisfies interpolating conditions, we can consider the corresponding problem for the function $f^{p / 2}$ in $F^{2}$. We leave the details to the reader. 


\section{REFERENCES}

1. D. Aharonov, C. Bénéteau, D. Khavinson, and H.S. Shapiro, "Extremal problems for nonvanishing functions in Bergman spaces", Selected Topics in Complex Analysis, 59-86, Oper. Theory Adv. Appl., 158, Birkhäuser, Basel, 2005.

2. V. Bargmann, "On a Hilbert space of analytic functions and an associated integral transform", Comm. Pure Appl. Math. 14 (1961) 187-214.

3. R. P. Boas, Entire Functions, Academic Press, New York, 1954.

4. P. Duren, D. Khavinson, H.S. Shapiro, and C. Sundberg, "Contractive zero-divisors in Bergman spaces", Pacific J. Math. 157 (1993), no. 1, 37-56.

5. P. Duren, D. Khavinson, H.S. Shapiro, and C. Sundberg, "Invariant subspaces in Bergman spaces and the biharmonic equation", Michigan Math. J. 41 (1994), no. 2, 247-259.

6. P. Duren and A. Schuster, Bergman Spaces, American Mathematical Society, Providence, R.I., 2004.

7. E. Fischer, "Über algebraische Modulsysteme und lineare homogene partielle Differentialgleichungen mit konstanten Koeffizienten", J. Für Math. 140 (1911), 48-81.

8. V. Fock, "Verallgemeinerung und Lösung der Diracschen statistischen Gleichung", Z. Physik 49 (1928), 339-357.

9. K. Guo and and D. Zheng, "Invariant Suspaces, quasi-invariant subspaces and Hankel operators", J. Funct. Anal. 187 (2001), 308-342.

10. H. Hedenmalm, "A factorization theorem for square area-integrable analytic functions", J. Reine Angew. Math. 422 (1991), 45-68.

11. H. Hedenmalm, B. Korenblum, and K. Zhu Theory of Bergman Spaces, Springer-Verlag, New York, 2000.

12. S. Kouchekian, "The density problem for unbounded Bergman operators", Integral Equations and Operator Theory 45 (2003), 319-342.

13. M. Lapidus and M. Von Frankenhuysen, "Complex Dimensions of Self-Similar Fractal Strings and Diophantine Approximation", Experiment. Math. 12 (2003), no. 1, 41-69.

14. A. I. Markushevich, Theory of functions of a complex variable. Vol. I, II, III, translated and edited by Richard A. Silverman, 2nd English edition, Chelsea Publishing Co., New York, 1977.

15. D. Newman and H. S. Shapiro, "A Hilbert Space of Entire Functions Related to the Operational Calculus", Mimeographed Notes, 1964.

16. D. Newman and H.S. Shapiro, "Fischer spaces of entire functions", Entire Functions and Related Parts of Analysis, pp. 360-369, Amer. Math. Soc., Providence, R.I., 1968.

17. K. Seip, "Density Theorems for sampling and interpolation in the Bargmann-Fock space I", J. Reine. Angew. Math. 429 (1992), 91-106.

18. K. Seip and R. Wallstén, "Density theorems for sampling and interpolation in the Bargmann-Fock space II", J. Reine. Angew. Math. 429 (1992), 107-113.

19. J. Tung, "Fock Spaces", Ph.D. Thesis, University of Michigan, 2005.

20. J. Tung, "Zero sets and interpolating sets in Fock spaces", Proc. Amer. Math. Soc. 134 (2006), no. 1, 259-263.

21. J. Tung, "Taylor coefficients of functions in Fock spaces", J. Math. Anal. Appl. 318 (2006), no. 2, 397-409.

22. J. Tung, "On Taylor coefficients and multipliers in Fock spaces", Banach spaces of analytic functions, 135-147, Contemp. Math. 454, Amer. Math. Soc., Providence, RI, 2008.

23. K. Zhu, "Zeros of Functions in Fock Spaces", Complex Variables 21 (1993), 87-98. 
Department of Mathematics and Statistics, University of South Florida, 4202 E. Fowler Ave., PHY114, Tampa, FL 33620

E-mail address: cbenetea@cas.usf.edu

Mathematics Department, Allegheny College, 520 N. Main St., Meadville PA 16335

E-mail address: brent.carswell@allegheny.edu

Department of Mathematics and Statistics, University of South Florida, 4202 E. Fowler Ave., PHY114, Tampa FL 33620

E-mail address: skouchek@cas.usf.edu 